\title{
On the Symmetry and Degeneracy of $\mathrm{H}_{3}^{+}$
}

\author{
Kyle N. Crabtree*,† and Benjamin J. McCall*, \\ ${ }^{\dagger}$ Harvard-Smithsonian Center for Astrophysics, 60 Garden Street, Cambridge, Massachusetts 02138, United States \\ ${ }^{\ddagger}$ Departments of Chemistry, Astronomy, and Physics, University of Illinois, 600 South Mathews Avenue, Urbana, Illinois 61801, \\ United States
}

ABSTRACT: The fundamental molecular ion $\mathrm{H}_{3}^{+}$has impacted astronomy, chemistry, and physics, particularly since the discovery of its rovibrational spectrum. Consisting of three identical fermions, its properties are profoundly influenced by the requirements of exchange symmetry, most notably the nonexistence of its ground rotational state. Spectroscopy of $\mathrm{H}_{3}^{+}$is often used to infer the relative abundances of its two nuclear spin modifications, ortho- and para- $\mathrm{H}_{3}^{+}$, which are important in areas as diverse as electron dissociative recombination and deuterium fractionation in cold interstellar

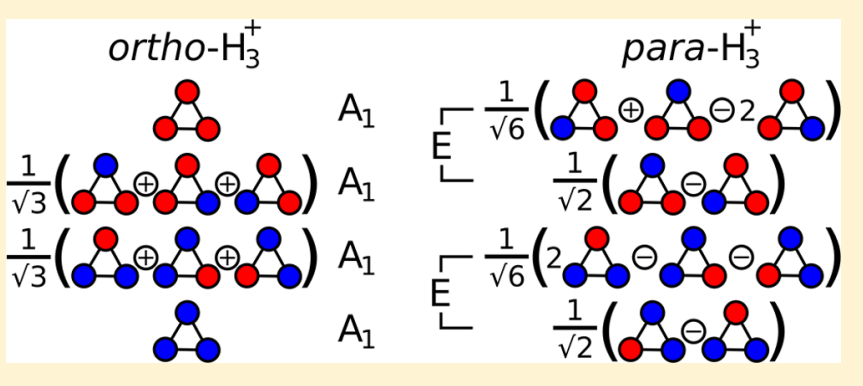
clouds. In this paper, we explore in detail the impact of exchange symmetry on the states of $\mathrm{H}_{3}^{+}$, with a particular focus on the state degeneracies necessary for converting spectral transition intensities to relative abundances. We address points of confusion in the literature surrounding these issues and discuss the implications for proton-transfer reactions of $\mathrm{H}_{3}^{+}$at low temperatures.

\section{INTRODUCTION}

The high-resolution spectrum of $\mathrm{H}_{3}^{+}$, first observed by Oka in $1980,{ }^{1}$ has served as a useful tool for probing fundamental chemical physics. As the simplest polyatomic molecule, $\mathrm{H}_{3}^{+}$serves as a useful benchmark for quantum chemistry, and its extensive laboratory spectroscopy ${ }^{2}$ (especially above the barrier to linearity $^{3-7}$ ) has helped spur advances in ab initio methods ${ }^{8-11}$ that now calculate $\mathrm{H}_{3}^{+}$energy levels to within $\sim 0.1 \mathrm{~cm}^{-1}$ of experimental accuracy, even at high energies. ${ }^{12,13}$ The dissociative recombination (DR) of $\mathrm{H}_{3}^{+}$with an electron is the simplest possible polyatomic DR process, ${ }^{14,15}$ and highresolution spectroscopy has helped to address the ongoing fundamental questions about its nuclear-spin-dependent recombination rates. $^{16-23}$

Spectroscopy of $\mathrm{H}_{3}^{+}$has also profoundly impacted the astrophysics community. $\mathrm{H}_{3}^{+}$had long been known to be the initiator of ion-molecule chemistry in the interstellar medium, ${ }^{24,25}$ but the infrared spectrum was ultimately what enabled its detection in the interstellar medium ${ }^{26-28}$ as well as in planetary atmospheres. ${ }^{29-31}$ In interstellar clouds, $\mathrm{H}_{3}^{+}$is measured in absorption, facilitating direct estimates of its abundance, and owing to its simple chemistry, the abundance can be related to the cosmic ray ionization rate in a direct fashion. Using this method, subsequent observations of $\mathrm{H}_{3}^{+}$have established that the cosmic ray ionization rate in diffuse molecular clouds is an order of magnitude greater than previously thought. ${ }^{32,33} \mathrm{H}_{3}^{+}$is also seen in sight lines toward the Galactic center, where it is used as a probe of temperature and density. ${ }^{34,35}$

A particularly fascinating example at the intersection of chemical physics, astrophysics, and high-resolution spectroscopy is the case of the $\mathrm{H}_{3}^{+}+\mathrm{H}_{2} \rightarrow \mathrm{H}_{2}+\mathrm{H}_{3}^{+}$proton scrambling reaction. From a chemical physics standpoint, this reaction exhibits selection rules based on the symmetries of the reactants' rovibronic and nuclear spin state, ${ }^{36}$ or alternatively, the selection rules can be derived in terms of nuclear spin angular momentum algebra. ${ }^{37}$ Because of the weakness of the nuclear magnetic interaction, the two nuclear spin modifications $\left(o-\mathrm{H}_{3}^{+}\right.$, angular momentum $I=(3 / 2)$, symmetry $\left.\Gamma_{\mathrm{ns}}=A_{1} ; p-\mathrm{H}_{3}^{+}, I=(1 / 2), \Gamma_{\mathrm{ns}}=E\right)$ can be regarded as separate chemical species; interconversion can only take place by means of reactive collisions or by interaction with a strong inhomogeneous magnetic field. As such, the $\mathrm{H}_{3}^{+}+\mathrm{H}_{2} \rightarrow$ $\mathrm{H}_{2}+\mathrm{H}_{3}^{+}$reaction has seen considerable theoretical treatment, especially in recent years, ${ }^{38-41}$ and the effects of the nuclear spin selection rules have been experimentally studied by highresolution spectroscopy of $\mathrm{H}_{3}^{+}$in hydrogenic plasmas. ${ }^{42-44}$ However, beyond the fundamentally intriguing chemical physics, this reaction is likely the most commonly occurring bimolecular reaction in the Universe. It plays a key role in establishing the "nuclear spin temperature" of $\mathrm{H}_{3}^{+}$in diffuse molecular clouds, ${ }^{45}$ and the partially deuterium-substituted versions of the $\mathrm{H}_{3}^{+}+\mathrm{H}_{2}$ reaction have the greatest influence on deuterium fractionation in dense clouds and prestellar cores. ${ }^{46-48}$

The applications mentioned above involve spectroscopically measuring the relative abundances of the two nuclear spin modifications of $\mathrm{H}_{3}^{+}$. Being a system composed of three indistinguishable nuclei, under the Born-Oppenheimer approximation, the nuclear Hamiltonian for $\mathrm{H}_{3}^{+}$must be invariant with respect to any permutation of the nuclei. Furthermore, because the nuclei in question are fermions, Fermi-Dirac statistics apply,

Special Issue: Oka Festschrift: Celebrating 45 Years of Astrochemistry

Received: January 3, 2013

Revised: $\quad$ March 27, 2013

Published: March 28, 2013 
and the total molecular wave function must be antisymmetric under pairwise permutations. In the framework of permutationinversion group theory, ${ }^{49} \mathrm{H}_{3}^{+}$belongs to the $S_{3}^{*}$ molecular symmetry group (see Table 1). For the majority of this work, the inversion symmetry (and corresponding parity labels on the representations) is not needed; therefore, for simplicity, the $S_{3}$ permutation group will be used instead; this is also shown in Table $1 .{ }^{50}$ In group theoretical terms, the requirement placed on

\section{Table $1 . S_{3}$ and $S_{3}^{*}$ Character Tables}

\begin{tabular}{|c|c|c|c|c|c|c|}
\hline \multicolumn{2}{|c|}{$S_{3}$} & \multicolumn{2}{|c|}{$E$} & $2(123)$ & \multicolumn{2}{|r|}{$3(12)$} \\
\hline & & 1 & & 1 & & 1 \\
\hline & & 1 & & 1 & & -1 \\
\hline & & 2 & & -1 & & 0 \\
\hline$S_{3}^{*}$ & $E$ & $2(123)$ & $3(12)$ & $E^{*}$ & $2(123)^{*}$ & $3(12)^{*}$ \\
\hline$A_{1}^{\prime}$ & 1 & 1 & 1 & 1 & 1 & 1 \\
\hline$A_{2}^{\prime}$ & 1 & 1 & -1 & 1 & 1 & -1 \\
\hline$E^{\prime}$ & 2 & -1 & 0 & 2 & -1 & 0 \\
\hline$A_{1}^{\prime \prime}$ & 1 & 1 & 1 & -1 & -1 & -1 \\
\hline$A_{2}^{\prime \prime}$ & 1 & 1 & -1 & -1 & -1 & 1 \\
\hline$E^{\prime \prime}$ & 2 & -1 & 0 & -2 & 1 & 0 \\
\hline
\end{tabular}

the total wave function by Fermi-Dirac statistics is that its total symmetry $\Gamma_{\text {tot }}$ must be $A_{2}$ as this is the only irreducible representation (irrep) under $S_{3}$ whose character is -1 with respect to all odd permutation cycles and +1 to all even cycles. Considering again the weakness of the nuclear magnetic interaction, the nuclear spin wave function can be treated separately from the rovibrational wave function, and the strict symmetry requirement is that $\Gamma_{\text {tot }}=A_{2} \subseteq \Gamma_{\mathrm{rv}} \otimes \Gamma_{\mathrm{ns}}{ }^{51}$ By inspection of the $S_{3}$ multiplication table (Table 2), it is apparent

Table 2. $S_{3}$ Multiplication Table

\begin{tabular}{llll}
$S_{3}$ & $A_{1}$ & $A_{2}$ & $E$ \\
$A_{1}$ & $A_{1}$ & $A_{2}$ & $E$ \\
$A_{2}$ & $A_{2}$ & $A_{1}$ & $E$ \\
$E$ & $E$ & $E$ & $A_{1} \oplus A_{2} \oplus E$ \\
\hline
\end{tabular}

that only certain combinations of nuclear spin and rovibrational states give the required symmetry, that is, $o-\mathrm{H}_{3}^{+}$and $p-\mathrm{H}_{3}^{+}$ correspond to different rovibrational states. It is this property that allows the abundances of the nuclear spin modifications to be measured using high-resolution spectroscopy.

While the relationship between the nuclear spin and rovibrational wave functions enables quantification of $o-\mathrm{H}_{3}^{+}$and $p-\mathrm{H}_{3}^{+}$from spectroscopic measurements, it also introduces subtleties that need to be carefully respected. The fundamental relationship in absorption spectroscopy, which is generally employed for these types of measurements, is the Beer-Lambert law, $I_{\mathrm{t}}(\nu)=I_{0}(\nu) \exp [-\sigma(\nu) n L]$, where $I_{0}(\nu)$ is the intensity of the incident radiation field at frequency $\nu$ and $I_{\mathrm{t}}$ is the intensity of the radiation transmitted after passage through a sample of the molecule of interest of length $L$ and number density $n$ and whose absorption cross section is $\sigma$. As $I_{\mathrm{t}}, I_{0}$, and $\sigma$ are all frequencydependent, integration over all frequencies is required to rigorously determine the value $n$ (assuming $\sigma$ and $L$ are known). From a practical standpoint, measuring the spectrum of a molecule at all frequencies is nearly impossible, and the task is often impractical even if only the frequencies at which the molecule has detectable absorption are considered. Thus, in the vast majority of cases, approximations and/or assumptions must be made to infer the total number density of a molecule given observations of a limited number of spectroscopic transitions; often, local thermodynamic equilibrium is invoked, and a Boltzmann distribution of the molecule's population among its states is assumed. However, for $\mathrm{H}_{3}^{+}$, frequently, the $o-\mathrm{H}_{3}^{+} / p-\mathrm{H}_{3}^{+}$ ratio is inconsistent with a Boltzmann distribution, and special care is required to properly account for this fact when interpreting a spectrum.

In this paper, the combinations of the nuclear spin wave functions of $\mathrm{H}_{3}^{+}$with its rovibrational wave functions are discussed in detail. The effects of exchange symmetry on the nature of the total wave function, particularly in regard to the degeneracies of the states, are enumerated by examining the forms of transformation matrices in the $S_{3}$ group. First, the nuclear spin states of $\mathrm{H}_{3}^{+}$are expressed in terms of a coupled angular momentum basis, and then the rovibrational states are expressed in terms of a basis of symmetric top wave functions with vibrational angular momenta. A direct product basis between the nuclear spin and rovibrational states is constructed, and linear combinations that satisfy exchange symmetry are explicitly found. Finally, the results of these calculations are used to examine aspects of the symmetry and degeneracy of $\mathrm{H}_{3}^{+}$states that pertain to experimental spectroscopy and chemical physics in three areas: its high-temperature statistical weights, its "nuclear spin temperature", and its proton-transfer reactions at low temperature. From the results of these calculations, it is inferred that the $\mathrm{H}_{2}$ molecules left behind in proton-transfer reactions of ground-state $p-\mathrm{H}_{3}^{+}$will have distribution of $1: 1$ ortho/para- $\mathrm{H}_{2}$. This is an important result for studies of deuterium fractionation in the interstellar medium, where only the lowest-energy states of $o-\mathrm{H}_{3}^{+}$and $p-\mathrm{H}_{3}^{+}$are populated.

\section{SYMMETRY OF $\mathrm{H}_{3}^{+}$}

2.1. Nuclear Spin. The goal of this section is to derive the nuclear spin wave functions for $o-\mathrm{H}_{3}^{+}$and $p-\mathrm{H}_{3}^{+}$. Because the proton is a spin- $(1 / 2)$ fermion, there are $(2 \times(1 / 2)+1)^{3}=8$ possible ways to couple the three spins in $\mathrm{H}_{3}^{+}$. The symmetry properties of the resultant combinations can be quickly derived from group theory as the character $\chi[\mathbf{R}]$ of the total reducible representation of $n$ particles of spin $i$ under the permutation operation $\mathbf{R}$ is $\chi[\mathbf{R}]=(2 i+1)^{n-x}$, where $x$ is the number of pairwise permutations (2-cycles) in operation $\mathbf{R}$. For a system of three spin- $(1 / 2)$ particles in the $S_{3}$ group, the reducible representation of all combinations $\Gamma_{\mathrm{r}}$ has the characters $\chi[E]=8$, $\chi[(12)]=4$, and $\chi[(123)]=2$, and using standard orthogonality relationships in group theory, the irreps are found to be $\Gamma_{\mathrm{r}}=4 A_{1}$ $\oplus 2 E$. This result implies that there are four $\mathrm{H}_{3}^{+}$nuclear spin wave functions that are totally symmetric under the operations of the $S_{3}$ group and also two pairs of wave functions that each transform together as $E$ (note that $E$ is a two-dimensional representation). Thus, there are four wave functions of each type; the $4 A_{1}$ wave functions are defined as $o-\mathrm{H}_{3}^{+}$, and the $2 \mathrm{E}$ wave functions are defined as $p-\mathrm{H}_{3}^{+}$, despite the fact that the labels in this case are arbitrary.

There are multiple ways to construct a coupled nuclear spin basis set from an uncoupled basis; here, we use angular momentum coupling. ${ }^{52}$ In this framework, the uncoupled basis consists of kets $\left|I_{a}, m_{a}\right\rangle$, where $I_{a}$ is the total nuclear spin angular momentum of particle $a$ and $m_{a}$ is its projection onto a chosen axis. $\mathrm{H}_{3}^{+}$is represented as three such particles, and as all have $I=(1 / 2)$, the values of $I$ are omitted for brevity, and the uncoupled basis consists of the three projections $\left|m_{a}, m_{b}, m_{c}\right\rangle$ onto 
some mutually chosen axis. To construct the coupled basis, the angular momentum of one pair of particles is first coupled, and then their coupled angular momentum is then coupled to the remaining particle. The coupling coefficients are ClebschGordan coefficients $\left\langle I_{x}, m_{x} ; I_{y}, m_{y} \mid I_{z}, m_{z}\right\rangle$, where $m_{z}=m_{x}+m_{y}$ and $I_{z}$ can take the values $\left\{I_{x}+I_{y},\left|I_{x}+I_{y}-1\right|, \ldots,\left|I_{x}-I_{y}\right|\right\}$. In the end, the six quantum numbers that specify the coupled basis states are $\left|I_{3}, m_{3}, I_{2}, I_{a}, I_{b}, I_{c}\right\rangle$, where $I_{3}$ is the total angular momentum of the three-particle system, $m_{3}$ its projection, and $I_{2}$ the coupled angular momentum of two arbitrarily chosen particles (for $\mathrm{H}_{3}^{+}$, this can be 0 or 1). Again, like in the uncoupled basis, $I_{a}, I_{b}$, and $I_{c}$ are all $(1 / 2)$ and are omitted for brevity. Mathematically, the coupled basis states are calculated by

$$
\begin{aligned}
\left|I_{3}, m_{3}, I_{2}\right\rangle= & \sum_{m_{a}, m_{b}, m_{c}}\left\langle I_{c}, m_{c} ; I_{2}, m_{2} \mid I_{3}, m_{3}\right\rangle\left\langle I_{a}, m_{a} ; I_{b}, m_{b} \mid I_{2}, m_{2}\right\rangle \\
& \times\left|m_{a}, m_{b}, m_{c}\right\rangle
\end{aligned}
$$

where $m_{2}=m_{a}+m_{b}$ as $I_{2}$ has been chosen as the coupled angular momentum of protons $a$ and $b$ (the choice is arbitrary as the labels $a, b$, and $c$ can be swapped without physical consequence). The results are listed in Table 3, where $\uparrow$ and $\downarrow$ are used to

\section{Table 3. Coupled Nuclear Spin Wave Functions of $\mathbf{H}_{3}^{+}$}

\begin{tabular}{lll}
$\Gamma_{\text {ns }}$ & \multicolumn{1}{c}{ coupled basis } & \multicolumn{1}{c}{ uncoupled basis } \\
$A_{1}$ & $|(3 / 2),(3 / 2), 1\rangle$ & $|\uparrow \uparrow \uparrow\rangle$ \\
$A_{1}$ & $|(3 / 2),(1 / 2), 1\rangle$ & $(1 / \sqrt{ } 3)(|\uparrow \uparrow \downarrow\rangle+|\uparrow \downarrow \uparrow\rangle+|\downarrow \uparrow \uparrow\rangle)$ \\
$A_{1}$ & $|(3 / 2),-(1 / 2), 1\rangle$ & $(1 / \sqrt{ } 3)(|\uparrow \downarrow \downarrow\rangle+|\downarrow \uparrow \downarrow\rangle+|\downarrow \downarrow \uparrow\rangle)$ \\
$A_{1}$ & $|(3 / 2),-(3 / 2), 1\rangle$ & $|\downarrow \downarrow \downarrow\rangle$ \\
$E$ & $|(1 / 2),(1 / 2), 1\rangle$ & $(1 / \sqrt{ } 6)(|\downarrow \uparrow \uparrow\rangle+|\uparrow \downarrow \uparrow\rangle-2|\uparrow \uparrow \downarrow\rangle)$ \\
& $|(1 / 2),(1 / 2), 0\rangle$ & $(1 / \sqrt{ } 2)(|\uparrow \downarrow \uparrow\rangle-|\downarrow \uparrow \uparrow\rangle)$ \\
$E$ & $|(1 / 2),-(1 / 2), 1\rangle$ & $(1 / \sqrt{ } 6)(2|\downarrow \downarrow \uparrow\rangle-|\downarrow \uparrow \downarrow\rangle-|\uparrow \downarrow \downarrow\rangle)$ \\
& $|(1 / 2),-(1 / 2), 0\rangle$ & $(1 / \sqrt{ } 2)(|\uparrow \downarrow \downarrow\rangle-|\downarrow \uparrow \downarrow\rangle)$ \\
\end{tabular}

represent projections of $+(1 / 2)$ and $-(1 / 2)$, respectively, in the uncoupled basis.

As can be seen by inspection, the four wave functions with $A_{1}$ symmetry all have $I_{3}=(3 / 2)$, and correspond to $o-\mathrm{H}_{3}^{+}$. The remaining coupled wave functions are grouped into pairs having $E$ symmetry; these all have $I_{3}=(1 / 2)$ and correspond to $p-\mathrm{H}_{3}^{+}$. While the $E$ symmetry may not be apparent by inspection, it can be confirmed by writing out explicitly the transformation matrices for the permutation operators in the $S_{3}$ group. The matrix elements for operator $\mathbf{R}$ are calculated as $\left\langle I_{3}^{\prime}, m_{3}^{\prime}, I_{2}^{\prime}|\mathbf{R}| I_{3}, m_{3}, I_{2}\right\rangle$. The operators $\mathbf{R}$ act directly on the

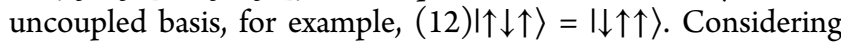
just the four $I_{3}=(1 / 2)$ wave functions, the transformation matrices for all $S_{3}$ operators (except $E$, which is the identity matrix) are

$$
(123)=\left(\begin{array}{cccc}
-\frac{1}{2} & \frac{\sqrt{3}}{2} & 0 & 0 \\
-\frac{\sqrt{3}}{2} & -\frac{1}{2} & 0 & 0 \\
0 & 0 & -\frac{1}{2} & \frac{\sqrt{3}}{2} \\
0 & 0 & -\frac{\sqrt{3}}{2} & -\frac{1}{2}
\end{array}\right)
$$

$$
(132)=\left(\begin{array}{cccc}
-\frac{1}{2} & -\frac{\sqrt{3}}{2} & 0 & 0 \\
\frac{\sqrt{3}}{2} & -\frac{1}{2} & 0 & 0 \\
0 & 0 & -\frac{1}{2} & -\frac{\sqrt{3}}{2} \\
0 & 0 & \frac{\sqrt{3}}{2} & -\frac{1}{2}
\end{array}\right)
$$$$
(12)=\left(\begin{array}{llll}
1 & 0 & 0 & 0 \\
0 & -1 & 0 & 0 \\
0 & 0 & 1 & 0 \\
0 & 0 & 0 & -1
\end{array}\right)
$$$$
(23)=\left(\begin{array}{cccc}
-\frac{1}{2} & -\frac{\sqrt{3}}{2} & 0 & 0 \\
-\frac{\sqrt{3}}{2} & \frac{1}{2} & 0 & 0 \\
0 & 0 & -\frac{1}{2} & -\frac{\sqrt{3}}{2} \\
0 & 0 & -\frac{\sqrt{3}}{2} & \frac{1}{2}
\end{array}\right)
$$$$
(13)=\left(\begin{array}{cccc}
-\frac{1}{2} & \frac{\sqrt{3}}{2} & 0 & 0 \\
\frac{\sqrt{3}}{2} & \frac{1}{2} & 0 & 0 \\
0 & 0 & -\frac{1}{2} & \frac{\sqrt{3}}{2} \\
0 & 0 & \frac{\sqrt{3}}{2} & \frac{1}{2}
\end{array}\right)
$$

The ordering of the rows and columns of these matrices are the same as the ordering of the wave functions in Table 3, that is, the basis states are unit vectors in the space

$$
\left(\begin{array}{l}
\left|\frac{1}{2}, \frac{1}{2}, 1\right\rangle \\
\left|\frac{1}{2}, \frac{1}{2}, 0\right\rangle \\
\left|\frac{1}{2},-\frac{1}{2}, 1\right\rangle \\
\left|\frac{1}{2},-\frac{1}{2}, 0\right\rangle
\end{array}\right)
$$

As expected from the $2 E$ symmetry, these matrices can be broken into identical $2 \times 2$ matrices, and each such matrix has the appropriate trace (i.e., character) for the operation. For completeness, it can be seen by inspection that the four $o-\mathrm{H}_{3}^{+}$ wave functions each transform as the $1 \times 1$ identity matrix under all $\mathbf{R} \in S_{3}$. 
2.2. Rotation-Vibration. The symmetry properties of the rotation-vibration wave functions of $\mathrm{H}_{3}^{+}$have been presented in detail a number of times (for one example, see the Ph.D. thesis of $\mathrm{McCall}^{53}$ ); therefore, only the salient properties will be summarized here. Analysis of the internal coordinates of $\mathrm{H}_{3}^{+}$ shows that its vibrations consist of a totally symmetric stretch $\left(\nu_{1}\right.$, symmetry $A_{1}$ ) and a pair of degenerate antisymmetric stretches $\left(\nu_{2}, E\right)$. From a symmetry standpoint, the relevant quantum number is the projection of the total vibrational angular momentum $(l)$ of the degenerate modes onto the molecular axis, which, for a given number of quanta in $\nu_{2}$, can take values $\left\{\nu_{2}\right.$, $\left.\nu_{2}-2, \ldots,-\nu_{2}\right\}$. This vibrational angular momentum couples to the rotational angular momentum to determine the overall rovibrational symmetry.

As the equilibrium geometry of $\mathrm{H}_{3}^{+}$is an equilateral triangle, it is convenient to use a basis of symmetric top wave functions to represent its rotational structure. ${ }^{54}$ These are the kets $|J, k, m\rangle$, where $J$ is the total rotational angular momentum, $k$ is its projection onto the molecular axis, and $m$ its projection onto a space-fixed axis. Like all angular momentum projections, $k$ and $m$ can take integer values between $J$ and $-J$. In a field-free environment, the $m$ states for a given $J$ and $k$ are all degenerate and contribute only a $(2 J+1)$-fold degeneracy; thus, the symmetric top states can be represented just by $|J, k\rangle$.

As mentioned above, the vibrational angular momentum couples to the molecular rotation, and as a result, the total ket for a rovibrational state is $|J, k, l\rangle$. The symmetry representations are determined by converting the permutation-inversion operations from the $S_{3}^{*}$ group into equivalent rotation operators, calculating the traces of the transformation matrices of these operators in the $|J, k, l\rangle$ basis and reducing (for details, see the book by Bunker and Jensen ${ }^{49}$ ). For $\mathrm{H}_{3}^{+}$, the results of these calculations for states with $l=0$ are shown in Table 4; similar

Table 4. Irreducible Representations of Rotational Angular Momentum States with $l=0$

\begin{tabular}{ll}
$\quad$ & \multicolumn{1}{c}{$\Gamma_{\mathrm{rv}}$ in $S_{3}(l=0)^{a}$} \\
$6 n^{b}$ & $n\left(2 A_{1} \oplus 2 A_{2} \oplus 4 E\right) \oplus A_{1}$ \\
$6 n+1$ & $n\left(2 A_{1} \oplus 2 A_{2} \oplus 4 E\right) \oplus A_{2} \oplus E$ \\
$6 n+2$ & $n\left(2 A_{1} \oplus 2 A_{2} \oplus 4 E\right) \oplus A_{1} \oplus 2 E$ \\
$6 n+3$ & $n\left(2 A_{1} \oplus 2 A_{2} \oplus 4 E\right) \oplus A_{1} \oplus 2 A_{2} \oplus 2 E$ \\
$6 n+4$ & $n\left(2 A_{1} \oplus 2 A_{2} \oplus 4 E\right) \oplus 2 A_{1} \oplus A_{2} \oplus 3 E$ \\
$6 n+5$ & $n\left(2 A_{1} \oplus 2 A_{2} \oplus 4 E\right) \oplus A_{1} \oplus 2 A_{2} \oplus 4 E$
\end{tabular}

${ }^{a^{a}}$ The parity is ignored in $S_{3}$, but for a state $|J, k, l\rangle$, its parity in $S_{3}^{*}$ is even (') if $k$ is even and odd (") if $k$ is odd. ${ }^{b} n$ is a non-negative integer.

tables can be derived for $l \neq 0$. Further analysis ${ }^{53}$ shows that under symmetry operations, the quantity $k-l$ is important for determining the symmetry of the $|J, k, l\rangle$ state; therefore, this is given a quantum number $g \equiv k-l$. Several results arise:

-The parity of a state $|J, k, l\rangle$ in $S_{3}^{*}$ is even (') if $k$ is even and odd (") if $k$ is odd. Parity is important for deriving selection rules for transitions but not for nuclear spin applications. Therefore, without loss of generality, the irreps from $S_{3}$ will be used here for concision, but the parity of any state under $S_{3}^{*}$ is easily obtained by inspection of the value of $k$.

-Time reversal symmetry implies that the states $|J, k, l\rangle$ and $|J,-k,-l\rangle$ are degenerate; therefore, the unsigned quantum number $G \equiv|g|=|k-l|$ is often used to label both states together. $^{55}$
-The symmetry of $|J, k, l\rangle$ in $S_{3}$ can be determined from $G$ (see Table 5).

Table 5. Irreducible Representations of $\mathrm{H}_{3}^{+}$RotationVibration States $|J, k, l\rangle$ in the $S_{3}$ Permutation Group

\begin{tabular}{ll}
\multicolumn{1}{c}{$G$} & $\Gamma_{\mathrm{rv}}$ in $S_{3}$ \\
$0,(k=0=l), J$ even & $A_{1}$ \\
$0,(k=0=l), J$ odd & $A_{2}$ \\
$3 n,(k \neq 0 \neq l)$ & $A_{1} \oplus A_{2}$ \\
$3 n \pm 1$ & $E$ \\
\hline
\end{tabular}

Finally, the transformation matrices with elements $\left\langle J^{\prime}, k^{\prime}, l^{\prime} \mid \mathbf{R} J, k, l\right\rangle\left(\mathbf{R} \in S_{3}\right)$ are enumerated here. Instead of writing out large $(2 J+1)$-dimensional matrices for each value of $l$ for each operator $\mathbf{R}$, by exploiting the previously derived symmetry properties, they can be broken into $(J+1)$ identical two-dimensional matrices plus a one-dimensional matrix for $l=0$ and $2(J+1)$ identical two-dimensional matrices for each $|l| \neq 0$. The one-dimensional matrices arise when $k=l=0$, and they are simply $E=(1),(12)=(23)=(13)=\left((-1)^{J}\right)$, and $(123)=(132)=(1)$. The other matrices are two-dimensional in the basis defined by unit vectors in

$$
\left(\begin{array}{c}
|J, k, l\rangle \\
I J,-k,-l\rangle
\end{array}\right)
$$

and have the forms

$$
\begin{aligned}
& (12)=\left(\begin{array}{cc}
0 & (-1)^{J} \\
(-1)^{J} & 0
\end{array}\right) \\
& (23)=\left(\begin{array}{cc}
0 & (-1)^{J} \mathrm{e}^{-4 \pi \mathrm{i} g / 3} \\
(-1)^{J} \mathrm{e}^{4 \pi \mathrm{i} g / 3} & 0
\end{array}\right) \\
& (13)=\left(\begin{array}{cc}
0 & (-1)^{J} \mathrm{e}^{4 \pi \mathrm{i} g / 3} \\
(-1)^{J} \mathrm{e}^{-4 \pi \mathrm{i} g / 3} & 0
\end{array}\right) \\
& (123)=\left(\begin{array}{cc}
\mathrm{e}^{2 \pi \mathrm{i} g / 3} & 0 \\
0 & \mathrm{e}^{-2 \pi \mathrm{i} g / 3}
\end{array}\right) \\
& (132)=\left(\begin{array}{cc}
\mathrm{e}^{-2 \pi \mathrm{i} g / 3} & 0 \\
0 & \mathrm{e}^{2 \pi \mathrm{i} g / 3}
\end{array}\right)
\end{aligned}
$$

2.3. Total Nuclear Wave Functions. In the absence of observable spin-rotation coupling, the symmetry of the total nuclear wave function can be obtained simply from a direct product of the rovibrational wave functions with the nuclear spin wave functions. Inspection of the $S_{3}$ multiplication table (Table 2) shows that in order to obtain an $A_{2}$ representation and satisfy exchange symmetry, $A_{1}$ states must combine with $A_{2}$ states (and vice versa), while $E$ states must combine with other $E$ states. Thus, the nuclear spin modifications having $4 A_{1} \oplus 2 E$ symmetry combine with different rovibrational states.

2.3.1. $k=0=l$. This special case is the most straightforward of all as the transformation matrices of the rovibrational states are one-dimensional. The direct product of the $(2 J+1)|J, 0,0\rangle$ states with the nuclear spin wave functions gives $(2 J+1) \times 4$ states with 
$A_{1}$ symmetry if $J$ is even or $A_{2}$ symmetry if $J$ is odd and $(2 J+1) \times 2$ pairs of states with $E$ symmetry. This is easily observed by taking direct products of the transformation matrices presented in the previous sections. Under the requirements of exchange symmetry, only the $(2 J+1) \times 4 A_{2}$ states are allowed. Thus, only the $I=(3 / 2)$ nuclear spin wave functions with $A_{1}$ symmetry $\left(o-\mathrm{H}_{3}^{+}\right)$can combine with $|J, 0,0\rangle$ states and only when $J$ is odd, leading to the well-known missing rotational levels in the ground vibrational state of $\mathrm{H}_{3}^{+}$.

2.3.2. $g=3 n$. When $g=3 n(n \in \mathbb{Z})$, the exponential terms in the rovibrational transformation matrices all become unity. By taking the linear combinations $|J, k, l\rangle_{ \pm}=(|J, k, l\rangle \pm|J,-k,-l\rangle) / \sqrt{2}$, the (12)-class transformation matrices become

$$
(12)=(23)=(13)=\left(\begin{array}{cc}
(-1)^{J} & 0 \\
0 & -(-1)^{J}
\end{array}\right)
$$

while the (123)-class matrices remain the identity matrix. Thus, the $|J, k, l\rangle_{+}$and $|J, k, l\rangle_{-}$states each can be represented one-dimensionally. If $J$ is even, then these two states have $A_{1}$ and $A_{2}$ symmetry, respectively, and vice versa if $J$ is odd. The direct product of these rovibrational states with the nuclear spin states in this case is then as straightforward as the case above, but there are no missing rotational levels. Each $g=3 n$ state with $A_{2}$ symmetry can combine with the $4 o-\mathrm{H}_{3}^{+}$nuclear spin states to give $(2 J+1) \times 4$ states with the required total $A_{2}$ symmetry.

2.3.3. $g=3 n \pm 1$. As shown in Table 5 , rovibrational wave functions with $g=3 n \pm 1$ have $E$ symmetry under $S_{3}$, and therefore, these must combine with $E$ nuclear spin wave functions in order to satisfy exchange symmetry (see Table 2). For a given $E$ pair of rovibrational states $|J, k, l\rangle$ and $|J,-k,-l\rangle$, a total of eight possible combinations with $\left|(1 / 2), m_{3}, I_{2}\right\rangle$ states can be constructed. Under the $S_{3}$ group, these eight combinations form a representation that reduces as $E \otimes 2 E=$ $2 A_{1} \oplus 2 A_{2} \oplus 2 E$; therefore, only two of the eight combinations satisfy exchange symmetry.

To find the linear combinations with $A_{2}$ symmetry, we will construct a direct product basis of rovibrational states with $I_{3}=(1 / 2)$ nuclear spin states. Taking advantage of the fact that the $m_{3}=(1 / 2)$ nuclear spin wave functions transform exactly the same as those with $m_{3}=-(1 / 2)$, it is sufficient to consider products involving only one of the two $E$ pairs as the results for the other pair are identical. The direct product basis consists of unit vectors in the vector space

$$
\left(\begin{array}{c}
\left|J, k, l, \frac{1}{2}, \frac{1}{2}, 1\right\rangle \\
\left|J, k, l, \frac{1}{2}, \frac{1}{2}, 0\right\rangle \\
\left|J,-k,-l, \frac{1}{2}, \frac{1}{2}, 1\right\rangle \\
\left|J,-k,-l, \frac{1}{2}, \frac{1}{2}, 0\right\rangle
\end{array}\right)
$$

and the transformation matrices in this basis are simply the Kronecker products of the corresponding matrices shown previously. These matrices can be brought into block diagonal form consistent with a direct sum of $A_{1} \oplus A_{2} \oplus E$ irreps by a unitary change of basis.

Suitable basis states are found by applying symmetry projectors $^{49}$ to the direct product basis states. A symmetry projector for irrep $\Gamma_{i}$ has the form

$$
\hat{\mathbf{p}}^{\Gamma_{i}}=\frac{f_{i}}{h} \sum \chi(\mathbf{R}) \mathbf{R}
$$

where $f_{i}$ is the dimension of $\Gamma_{i}, h$ is the order of the group, $\mathbf{R}$ is an element of the group, $\chi(\mathbf{R})$ is the character of $\mathbf{R}$ in $\Gamma_{i}$, and the sum is over all elements in the group. The new basis consists of the linear combinations $\hat{\mathbf{p}}^{A_{1}}\left|J, k, l, \frac{1}{2}, \frac{1}{2}, 1\right\rangle, \hat{\mathbf{p}}^{A_{2}}\left|J, k, l, \frac{1}{2}, \frac{1}{2}, 1\right\rangle$, $\hat{\mathbf{p}}^{E}\left|J, k, l, \frac{1}{2}, \frac{1}{2}, 1\right\rangle$, and $\hat{\mathbf{p}}^{E}\left|J,-k,-l, \frac{1}{2}, \frac{1}{2}, 1\right\rangle$, and they are shown explicitly in the top half of Table $6 .^{56}$ The unitary changeof-basis matrix

$$
\mathbf{T}=\left(\begin{array}{cccc}
\frac{1}{2} & \frac{1}{2} & \frac{1}{\sqrt{2}} & 0 \\
\mp \frac{\mathrm{i}}{2} & \mp \frac{\mathrm{i}}{2} & \pm \frac{\mathrm{i}}{\sqrt{2}} & 0 \\
\frac{(-1)^{J}}{2} & \frac{-(-1)^{J}}{2} & 0 & \frac{1}{\sqrt{2}} \\
\pm \frac{\mathrm{i}(-1)^{J}}{2} & \mp \frac{\mathrm{i}(-1)^{J}}{2} & 0 & \pm \frac{\mathrm{i}}{\sqrt{2}}
\end{array}\right)
$$

where the signs are consistent with $g=3 n \pm 1$, allows conversion from the direct product basis to the symmetrized basis.

After applying the unitary change of basis $\mathbf{R}^{\prime}=\mathbf{T}^{\dagger} \mathbf{R} \mathbf{T}$, the transformation matrices in the new symmetrized basis $\mathbf{R}^{\prime}$ are brought into the appropriate block diagonal form

$$
\begin{aligned}
& (12)=\left(\begin{array}{cccc}
1 & 0 & 0 & 0 \\
0 & -1 & 0 & 0 \\
0 & 0 & 0 & (-1)^{J} \\
0 & 0 & (-1)^{J} & 0
\end{array}\right) \\
& (23)=\left(\begin{array}{cccc}
1 & 0 & 0 & 0 \\
0 & -1 & 0 & 0 \\
0 & 0 & 0 & -\frac{(-1)^{J} \mathrm{e}^{ \pm 2 \pi \mathrm{i} / 3}}{1+\mathrm{e}^{ \pm 2 \pi \mathrm{i} / 3}} \\
0 & 0 & -\frac{(-1)^{J} \mathrm{e}^{\mp 2 \pi \mathrm{i} / 3}}{1+\mathrm{e}^{\mp 2 \pi \mathrm{i} / 3}} & 0
\end{array}\right) \\
& (13)=\left(\begin{array}{cccc}
1 & 0 & 0 & 0 \\
0 & -1 & 0 & 0 \\
0 & 0 & 0 & -\frac{(-1)^{J} \mathrm{e}^{\mp 2 \pi \mathrm{i} / 3}}{1+\mathrm{e}^{\mp 2 \pi \mathrm{i} / 3}} \\
0 & 0 & -\frac{(-1)^{J} \mathrm{e}^{ \pm 2 \pi \mathrm{i} / 3}}{1+\mathrm{e}^{ \pm 2 \pi \mathrm{i} / 3}} & 0
\end{array}\right)
\end{aligned}
$$


Table 6. Linear Combinations of Direct Product Basis Functions that Transform as irreps of $S_{3}$, with $g=3 n \pm 1$ and $I_{3}=(1 / 2)$

\begin{tabular}{ll}
$\Gamma$ & \multicolumn{1}{c}{ linear combination } \\
$m_{3}=(1 / 2)$ & $\frac{1}{2}\left[\left|J, k, l, \frac{1}{2}, \frac{1}{2}, 1\right\rangle \mp \mathrm{i}\left|J, k, l, \frac{1}{2}, \frac{1}{2}, 0\right\rangle+(-1)^{J}\left|J,-k,-l, \frac{1}{2}, \frac{1}{2}, 1\right\rangle \pm \mathrm{i}(-1)^{J}\left|J,-k,-l, \frac{1}{2}, \frac{1}{2}, 0\right\rangle\right]$ \\
$A_{1}$ & $\frac{1}{2}\left[\left|J, k, l, \frac{1}{2}, \frac{1}{2}, 1\right\rangle \mp \mathrm{i}\left|J, k, l, \frac{1}{2}, \frac{1}{2}, 0\right\rangle-(-1)^{J}\left|J,-k,-l, \frac{1}{2}, \frac{1}{2}, 1\right\rangle \mp \mathrm{i}(-1)^{J}\left|J,-k,-l, \frac{1}{2}, \frac{1}{2}, 0\right\rangle\right]$ \\
$A_{2}$ & $\frac{1}{\sqrt{2}}\left[\left|J, k, l, \frac{1}{2}, \frac{1}{2}, 1\right\rangle \pm \mathrm{i}\left|J, k, l, \frac{1}{2}, \frac{1}{2}, 0\right\rangle\right]$ \\
$E$ & $\frac{1}{\sqrt{2}}\left[\left|J,-k,-l, \frac{1}{2}, \frac{1}{2}, 1\right\rangle \mp \mathrm{i}\left|J,-k,-l, \frac{1}{2}, \frac{1}{2}, 0\right\rangle\right]$ \\
$E$ & $\frac{1}{2}\left[\left|J, k, l, \frac{1}{2},-\frac{1}{2}, 1\right\rangle \mp \mathrm{i}\left|J, k, l, \frac{1}{2},-\frac{1}{2}, 0\right\rangle+(-1)^{J}\left|J,-k,-l, \frac{1}{2},-\frac{1}{2}, 1\right\rangle \pm \mathrm{i}(-1)^{J}\left|J,-k,-l, \frac{1}{2},-\frac{1}{2}, 0\right\rangle\right]$ \\
$m_{3}=-(1 / 2)$ & $\frac{1}{2}\left[\left|J, k, l, \frac{1}{2},-\frac{1}{2}, 1\right\rangle \mp \mathrm{i}\left|J, k, l, \frac{1}{2},-\frac{1}{2}, 0\right\rangle-(-1)^{J}\left|J,-k,-l, \frac{1}{2},-\frac{1}{2}, 1\right\rangle \mp \mathrm{i}(-1)^{J}\left|J,-k,-l, \frac{1}{2},-\frac{1}{2}, 0\right\rangle\right]$ \\
$A_{1}$ & $\frac{1}{\sqrt{2}}\left[\left|J, k, l, \frac{1}{2},-\frac{1}{2}, 1\right\rangle \pm \mathrm{i}\left|J, k, l, \frac{1}{2},-\frac{1}{2}, 0\right\rangle\right]$ \\
$A_{2}$ & $\frac{1}{\sqrt{2}}\left[\left|J,-k,-l, \frac{1}{2},-\frac{1}{2}, 1\right\rangle \mp \mathrm{i}\left|J,-k,-l, \frac{1}{2},-\frac{1}{2}, 0\right\rangle\right]$ \\
$E$ &
\end{tabular}

$$
\begin{aligned}
& (123)=\left(\begin{array}{cccc}
1 & 0 & 0 & 0 \\
0 & 1 & 0 & 0 \\
0 & 0 & -\frac{\mathrm{e}^{ \pm 2 \pi \mathrm{i} / 3}}{1+\mathrm{e}^{ \pm 2 \pi \mathrm{i} / 3}} & 0 \\
0 & 0 & 0 & \frac{1}{1+\mathrm{e}^{ \pm 2 \pi \mathrm{i} / 3}}
\end{array}\right) \\
& (132)=\left(\begin{array}{cccc}
1 & 0 & 0 & 0 \\
0 & 1 & 0 & 0 \\
0 & 0 & \frac{1}{1+\mathrm{e}^{ \pm 2 \pi \mathrm{i} / 3}} & 0 \\
0 & 0 & 0 & -\frac{\mathrm{e}^{ \pm 2 \pi \mathrm{i} / 3}}{1+\mathrm{e}^{ \pm 2 \pi \mathrm{i} / 3}}
\end{array}\right)
\end{aligned}
$$

Identical results are derived for the $E$ pair of nuclear spin basis states with $m_{3}=-(1 / 2)$ (see the bottom half of Table 6$)$. Thus, pairs of rovibrational wave functions with $g=3 n \pm 1$ combine with the four $I_{3}=(1 / 2)\left(p-\mathrm{H}_{3}^{+}\right)$nuclear spin states to give a total of two allowed states [ignoring the $(2 J+1)$-fold degeneracy].

\section{DISCUSSION}

Having expressed the nuclear wave function for $\mathrm{H}_{3}^{+}$in terms of linear combinations of rovibrational and nuclear spin basis states, we can now consider the implications of these results for the interpretation of experiments. We will focus on three aspects, the high-temperature statistical weights of $o-\mathrm{H}_{3}^{+}$and $p-\mathrm{H}_{3}^{+}$, the "spin temperature" of $\mathrm{H}_{3}^{+}$, and proton-transfer reactions at low temperature.

3.1. Statistical Weights. The statistical weights of $o-\mathrm{H}_{3}^{+}$and $p-\mathrm{H}_{3}^{+}$are important for the aforementioned proton scrambling reaction $\mathrm{H}_{3}^{+}+\mathrm{H}_{2} \rightarrow \mathrm{H}_{2}+\mathrm{H}_{3}^{+}$. As this reaction proceeds through a $\left(\mathrm{H}_{5}^{+}\right)^{*}$ collision complex, the protons in the reactant $\mathrm{H}_{3}^{+}$and $\mathrm{H}_{2}$ molecules can rearrange, in some cases resulting effectively in ortho-para conversion. In the high-temperature limit, the relative rates of the 16 possible reactions consisting of combinations of reactant and product pairs (e.g., $o-\mathrm{H}_{3}^{+}+p-\mathrm{H}_{2} \rightarrow o-\mathrm{H}_{2}+$ $p-\mathrm{H}_{3}^{+}$) can be inferred by simply counting the number of pathways connecting the reactants with their respective products. Thus, it is necessary to know how many $o-\mathrm{H}_{3}^{+}$states exist relative to $p-\mathrm{H}_{3}^{+}$ states.
It is well-known and experimentally observed that the ortho/ para ratio of $\mathrm{H}_{3}^{+}$at high temperature is $1: 1$; however, various different explanations for this ratio have been given. According to $\mathrm{Oka}^{37}$ the high-temperature statistical weights are simply proportional to the number of nuclear spin wave functions sharing a common total angular momentum, which is easily calculated using angular momentum algebra. For $\mathrm{H}_{3}^{+}$, $\mathcal{D}_{1 / 2}^{3}=\mathcal{D}_{3 / 2} \oplus 2 \mathcal{D}_{1 / 2}$, where $D_{I}$ is the rotation group representation of a nuclear spin angular momentum $D_{I}$ with degeneracy $2 I+1$. The coefficient in front of each term is the dimension of the representation, and thus the total number of nuclear spin wave functions of each type is the dimension times the degeneracy of each representation, yielding ortho/para $=4: 4$ for $\mathrm{H}_{3}^{+}$.

Hugo et al. ${ }^{39}$ instead explain that the statistical weights arise from the frequencies and dimensions of the irreps of the nuclear spin wave functions $\left(\Gamma_{\mathrm{ns}}\right)$ rather than from the rotation group representations of the nuclear spin angular momentum. The dimension of $\Gamma_{n s}$ is related to the high-temperature statistical weights because the number of rovibrational states having the same symmetry $\left(\Gamma_{\mathrm{rv}}=\Gamma_{\mathrm{ns}}\right)$ is proportional to the dimension of $\Gamma_{\mathrm{rv}}$. This can be verified for $\mathrm{H}_{3}^{+}$by inspection of Table 4; in the limit that $J$ is large, there are twice as many $E$ states as $A_{2}$ or $A_{1}$ states. It should be noted that this explanation is imprecise as the appropriate rovibrational states are not strictly those with the same symmetry as $\Gamma_{\mathrm{ns}}$ but rather those whose products contain the irrep that satisfies exchange symmetry (in the case that multiple representations satisfy exchange symmetry, as in methane, these must also be taken into account). For instance, for $o-\mathrm{H}_{3}^{+}\left(A_{1}\right)$, the appropriate rovibrational states are those with $A_{2}$ symmetry rather than $A_{1}$ as $A_{1} \otimes A_{2}=A_{2}$. Nevertheless, the results of Hugo et al. are correct for $\mathrm{H}_{3}^{+}, \Gamma_{\mathrm{ns}}=4 A_{1} \oplus 2 E$, and the same results are obtained for $o-\mathrm{H}_{3}^{+} / p-\mathrm{H}_{3}^{+},(4 \times 1):(2 \times 2)$.

The results in the present work are consistent with both of these explanations as there is a 1:1 mapping between nuclear spin angular momentum and symmetry irreps. Oka's method works for $\mathrm{H}_{3}^{+}$as he relies on the fact that the requirement of exchange symmetry automatically takes into account the number of rovibrational states with which the nuclear spin wave functions can combine, and this is borne out by the detailed explanation by Hugo et al. The situation becomes more interesting when the 1:1 mapping breaks down, as in $\mathrm{D}_{3}^{+}$. Angular momentum algebra gives $\left(\mathcal{D}_{1}\right)^{3}=\mathcal{D}_{3} \oplus 2 \mathcal{D}_{2} \oplus 3 \mathcal{D}_{1} \oplus \mathcal{D}_{0}$, while the symmetry 
irreps are $10 A_{1} \oplus 16 E \oplus A_{2}$. To first order, these can be brought into agreement by recognizing that the $3 \mathcal{D}_{1}$ rotation group representation breaks down into $3 A_{1} \oplus 3 E$, but it has been called into question ${ }^{57}$ whether nuclear spin angular momentum is a good quantum number in this context. Applying the methods in the present work to $\mathrm{D}_{3}^{+}$, while beyond the scope of this paper, may provide some insight for experiments that can test whether states with the same symmetry but different nuclear spin angular momentum can be distinguished.

3.2. Spin Temperature of $\mathrm{H}_{3}^{+}$. In astronomical studies of $\mathrm{H}_{3}^{+}$ in the interstellar medium, typically only rovibrational transitions in the infrared arising from the two lowest levels $[(J, G)=(1,1)$ and $(1,0)]$ in the $\nu_{2}$ fundamental band are observed, owing to the low temperatures and the faster rate of radiative relaxation relative to collisional pumping. ${ }^{58}$ From the values of the quantum number $G$ of these two states, it is evident that the ground state $(1,1)$ is a $p-\mathrm{H}_{3}^{+}$state, while the $(1,0)$ state, $\sim 32 \mathrm{~K}$ higher in energy, is $o-\mathrm{H}_{3}^{+}$. If, as is often assumed, ${ }^{27,28}$ the $\mathrm{H}_{3}^{+}+\mathrm{H}_{2} \rightarrow \mathrm{H}_{2}+\mathrm{H}_{3}^{+}$ reaction is fast enough to bring the ortho/para ratio into thermodynamic equilibrium with the background gas (though the validity of this assumption in at least some environments is questionable ${ }^{45}$ ), the observed spectrum can be used to infer the kinetic temperature of the cloud

$$
\frac{n_{10}}{n_{11}}=\frac{I_{10} \mu_{11}^{2}}{I_{11} \mu_{10}^{2}}=\frac{g_{10}}{g_{11}} \exp \left(\frac{-\Delta E}{T}\right)
$$

where the subscripts are the $J$ and $G$ quantum numbers of the state, $n$ the number density, $I$ the observed intensity of the transition in the spectrum, $\mu^{2}$ the transition dipole moment, $g$ the degeneracy of the state, and $\Delta E=\left(E_{10}-E_{11}\right) / k_{\mathrm{B}} \approx 32 \mathrm{~K}$. The quantity $T$ in this equation is the so-called spin temperature of $\mathrm{H}_{3}^{+}$, and it is assumed to be equal to the kinetic temperature.

Setting aside any questions regarding the applicability of this calculation for the physics of interstellar clouds, the aspect of this equation relevant for this paper is the ratio of state degeneracies, $g_{10} / g_{11}$. This quantity (or similar quantities) comes into play any time populations of $p-\mathrm{H}_{3}^{+}$and $o-\mathrm{H}_{3}^{+}$are compared. The correct answer, as is evident from the calculations above, is $12 / 6$, and indeed, this is the value used in the astronomical studies, ${ }^{27,28}$ though using recommendations from at least one other publication, ${ }^{5}$ the value $12 / 12$ would be inferred as the authors state that $\mathrm{H}_{3}^{+}$has a "strict double degeneracy for $G>0$ ". Application of this statement to the ground state would imply that the $(J, g)=(1,1)$ and $(1,-1)$ states contribute an additional factor of 2 to the degeneracy, and we assume that the authors intended to imply that the total degeneracy is given by $2 \times(2 J+1)(2 I+1)$ for all states with $G>0$ and $(2 J+1)(2 I+1)$ for states with $G=0$.

In section 2.3, we derived the linear combinations of rovibrational and nuclear spin states that satisfy the total symmetry requirement of $A_{2}$. To find the degeneracy of the groundstate wave function, we note that in the ground vibrational state, $l=0$, and therefore, the rovibrational kets $|1,1,0\rangle$ and $|1,-1,0\rangle$ are involved. Each of these kets can combine with the four $p-\mathrm{H}_{3}^{+}$ nuclear spin wave functions, giving a total of eight total nuclear wave functions, ignoring the $(2 J+1)$-fold degeneracy. However, of those eight wave functions, only two linear combinations have $A_{2}$ symmetry (see Table 6 , substituting $J=1, k=1, l=0$ ) and are the only allowed states. Thus, the $(J, G)=(1,1)$ state (and any $p-\mathrm{H}_{3}^{+}$state) has total degeneracy of $2 \times(2 J+1)$, where the factor of 2 takes into account the nuclear spin and $G$ degeneracies together, as opposed to $4 \times(2 J+1)$, which would be derived from the aforementioned publication. ${ }^{5}$ To complete the example, states with
$G>0$ and $G=3 n$ (corresponding to $o-\mathrm{H}_{3}^{+}$) have a total degeneracy of $4 \times(2 J+1)$ by the arguments discussed in section 2.3.2, while one would derive $2 \times(2 J+1)(2 I+1)=8 \times(2 J+1)$ under the assumption of a strict double degeneracy from $G>0$.

One further observation can be made related to the degeneracies of these states. The equation shown above implies that in the limit that $T \rightarrow 0, n_{10} / n_{11} \rightarrow 0$, and thus, the $o-\mathrm{H}_{3}^{+} / p-\mathrm{H}_{3}^{+}$ ratio should approach 0 (alternatively, the fraction of $p-\mathrm{H}_{3}^{+}, p_{3}$, approaches 1 ), while in the high-temperature limit, as discussed earlier, $p_{3} \rightarrow(1 / 2)$. A third limit, though, is given by the ratio of the degeneracies of the lowest two states, $p_{3} \rightarrow(1 / 3)\left(o-\mathrm{H}_{3}^{+} / p\right.$ $\left.\mathrm{H}_{3}^{+} \rightarrow 2\right)$. Such a situation could be achieved in an environment in which $o-\mathrm{H}_{3}^{+} \leftrightarrow p-\mathrm{H}_{3}^{+}$interconversion occurs freely, but only the two lowest states of $\mathrm{H}_{3}^{+}$can be significantly populated. This may be possible in a collisionally cold environment with excess $o-\mathrm{H}_{2}$, and it has been anticipated by kinetic models of the $\mathrm{H}_{3}^{+}+\mathrm{H}_{2}$ reaction at low temperature, ${ }^{40}$ though the authors did not focus on this aspect. An enhancement of $o-\mathrm{H}_{3}^{+}$above the high-temperature limit may be desirable for studies of nuclear-spin-dependent reaction rates of $\mathrm{H}_{3}^{+}$ (such as dissociative recombination).

Such an enhancement may have been observed in preliminary measurements reported by Kreckel et al. in a cold 22-pole ion trap. ${ }^{59}$ In this study, the authors attempted to manipulate the ortho/para ratio of $\mathrm{H}_{3}^{+}$by changing the ortho/para ratio of the $\mathrm{H}_{2}$ fed into a high-temperature discharge source and then cooling the $\mathrm{H}_{3}^{+}$ions with buffer gas inside of a 22-pole ion trap. Leakage of the $\mathrm{H}_{2}$ from the source into the trap caused $\mathrm{H}_{3}^{+}+\mathrm{H}_{2}$ nuclear spin interconversion reactions to occur, and the final ortho/para ratio of $\mathrm{H}_{3}^{+}$was found to be $60: 40\left(p_{3}=0.4\right)$ when normal $\mathrm{H}_{2}$ (ortho/ para $=3: 1)$ was used as the source gas. It is possible that at even lower temperatures, the value of $p_{3}$ would continue to approach the limiting value of $(1 / 3)$.

3.3. Proton-Transfer Reactions. The proclivity of $\mathrm{H}_{3}^{+}$to transfer one of its protons to another molecule, combined with its ready formation in space, makes it one of the most important molecules in interstellar chemistry. ${ }^{24,25}$ An aspect of the protontransfer process rarely considered is the nuclear spin modification of the $\mathrm{H}_{2}$ molecule left behind after the protontransfer reaction has occurred. Particularly in colder, denser regions of space, the ortho/para ratio of $\mathrm{H}_{2}$ has a strong influence on deuterium fractionation, ${ }^{47,48}$ and a significant fraction of the total $\mathrm{H}_{2}$ population is generated from proton-transfer reactions of $\mathrm{H}_{3}^{+}$. Thus, it is appropriate to consider the branching fractions of these proton-transfer reactions, as have been discussed by Widicus Weaver et al. in the case of $\mathrm{H}_{3}^{+}+\mathrm{O}_{2} \rightarrow \mathrm{HO}_{2}^{+}+\mathrm{H}_{2}{ }^{60}$

We will assume a general reaction $\mathrm{H}_{3}^{+}+\mathrm{X} \rightarrow \mathrm{HX}^{+}+\mathrm{H}_{2}$ whose mechanism is a direct proton hop (i.e., no complex is formed that can result in proton scrambling prior to dissociation). When the reaction involves $o-\mathrm{H}_{3}^{+}$, application of Oka's angular momentum algebra ${ }^{37}$ quickly shows that only $o-\mathrm{H}_{2}$ can be formed as formation of $p-\mathrm{H}_{2}$ would violate conservation of total nuclear spin angular momentum. Although the angular momentum algebra only gives proper branching ratios in the high-temperature limit, this selection rule for the chemical reaction also applies at the low temperatures relevant for cold interstellar clouds. However, we can alternatively derive this result by inspecting the $o-\mathrm{H}_{3}^{+}$nuclear spin wave functions. In this approach, we designate proton $c$ (in the uncoupled basis) as the proton that is transferred in the reaction, and this means that the $I_{2}$ quantum number is the coupled angular momentum of the two protons that constitute the resultant $\mathrm{H}_{2}$ product molecule. Proton $c$ is chosen to be consistent with the wave functions in Table 3; equivalent results can be derived using protons $a$ or $b$ by altering the angular 
momentum coupling scheme used to derive the nuclear spin wave functions. In all four $o-\mathrm{H}_{3}^{+}$wave functions, $I_{2}=1$, and therefore, we obtain the same result as the angular momentum algebra for the branching fraction, but this result applies to every individual $o-\mathrm{H}_{3}^{+}$state.

With $p-\mathrm{H}_{3}^{+}$, the result is more subtle. Angular momentum algebra shows that the branching fractions for the formation of $o-\mathrm{H}_{2}$ and $p-\mathrm{H}_{2}$ are equal in the high-temperature limit, but in interstellar space, only the lowest rotational state of $\mathrm{H}_{3}^{+}$is populated. Looking at the $p-\mathrm{H}_{3}^{+}$wave functions in Table 3 shows that two of the four have $I_{2}=1$, and the other two have $I_{2}=0$, which is consistent with the angular momentum algebra. However, using the results of the direct product basis, we can see exactly which nuclear spin wave functions contribute to the lowest state of $p-\mathrm{H}_{3}^{+}$; these are given by the $A_{2}$ linear combinations in Table 6 , substituting $J=1, g=1, k=1$, and $l=0$. By taking the magnitudes squared of the coefficients of those wave functions, the total probability amplitudes for $I_{2}=1$ and 0 are equal, implying that even for the lowest rotational state of $p-\mathrm{H}_{3}^{+}$, a proton-transfer reaction should have equal branching fractions for the formation of $o-\mathrm{H}_{2}$ and $p-\mathrm{H}_{2}$. Thus, proton-transfer reactions involving $\mathrm{H}_{3}^{+}$may lead to an enhancement of the $o-\mathrm{H}_{2} /$ $p-\mathrm{H}_{2}$ ratio in cold, dense interstellar clouds as these reactions produce a much larger fraction of $o-\mathrm{H}_{2}$ than would be expected from the kinetic temperature of the environment $\left(o-\mathrm{H}_{2} / p-\mathrm{H}_{2}\right.$ is $\sim 10^{-3}$ at $20 \mathrm{~K}$ ).

\section{CONCLUSIONS}

In this paper, we have derived the wave functions for $\mathrm{H}_{3}^{+}$ expressed in terms of linear combinations of direct products of symmetric top rotational basis states with vibrational angular momentum and coupled nuclear spin angular momentum basis states. These wave functions are valid in the limit that $J, k$, and $l$ are good quantum numbers and are only intended to capture the elements of the states that contribute to the total symmetry rather than to serve as an accurate description of the spatial amplitudes of the true molecular wave function. Exchange symmetry was taken into account by constructing a symmetrized basis from the direct product states. Under the new basis, the transformation matrices of the elements of the $S_{3}$ permutation group are of block diagonal form with the appropriate block dimensionalities and characters; the representations with $A_{2}$ symmetry satisfy exchange symmetry. Using the results of these calculations, we have evaluated aspects of the symmetry and degeneracy of $\mathrm{H}_{3}^{+}$(particularly $p-\mathrm{H}_{3}^{+}$) that have caused some confusion in the literature. We have also shown that by inspection of the $p-\mathrm{H}_{3}^{+}$nuclear spin wave functions that contribute to its lowest-energy state, proton-transfer reactions should to first order give branching fractions for formation of $o-\mathrm{H}_{2}$ and $p-\mathrm{H}_{2}$ consistent with the predictions of angular momentum algebra in the high-temperature limit.

\section{AUTHOR INFORMATION}

\section{Corresponding Author}

*E-mail: kcrabtree@cfa.harvard.edu (K.N.C.); bjmccall@illinois. edu (B.J.M.).

\section{Notes}

The authors declare no competing financial interest.

\section{ACKNOWLEDGMENTS}

We thank Takeshi Oka for inspiring us over the years with his enthusiasm for $\mathrm{H}_{3}^{+}$. K.N.C. has been supported by a CfA
Postdoctoral Fellowship from the Smithsonian Astrophysical Observatory.

\section{REFERENCES}

(1) Oka, T. Observation of the Infrared Spectrum of $\mathrm{H}_{3}^{+}$. Phys. Rev. Lett. 1980, $45,531-534$.

(2) Lindsay, C. M.; McCall, B. J. Comprehensive Evaluation and Compilation of $\mathrm{H}_{3}^{+}$Spectroscopy. J. Mol. Spectrosc. 2001, 210, 60-83.

(3) Gottfried, J. L.; McCall, B. J.; Oka, T. Near-Infrared Spectroscopy of $\mathrm{H}_{3}^{+}$above the Barrier to Linearity. J. Chem. Phys. 2003, 118, 1089010899.

(4) Gottfried, J. L. Near-Infrared Spectroscopy of $\mathrm{H}_{3}^{+}$above the Barrier to Linearity. Philos. Trans. R. Soc. London, Ser. A 2006, 364, 2917-2929.

(5) Kreckel, H.; Bing, D.; Reinhardt, S.; Petrignani, A.; Berg, M.; Wolf, A. Chemical Probing Spectroscopy of $\mathrm{H}_{3}^{+}$above the Barrier to Linearity. J. Chem. Phys. 2008, 129, 164312.

(6) Morong, C. P.; Gottfried, J. L.; Oka, T. $\mathrm{H}_{3}^{+}$as the Benchmark for Rigorous Ab Initio Theory. J. Mol. Spectrosc. 2009, 255, 13-23.

(7) Berg, M.; Wolf, A.; Petrignani, A. Visible Transitions from Ground State $\mathrm{H}_{3}^{+}$Measured with High-Sensitivity Action Spectroscopy. Philos. Trans. R. Soc. London, Ser. A 2012, 370, 5028-5040.

(8) Meyer, W.; Botschwina, P.; Burton, P. Ab Initio Calculation of Near-Equilibrium Potential and Multipole Moment Surfaces and Vibrational Frequencies of $\mathrm{H}_{3}^{+}$and Its Isotopomers. J. Chem. Phys. 1986, 84, 891-900.

(9) Tennyson, J.; Polyansky, O. L. Non-Born-Oppenheimer Correction to the $\mathrm{H}_{3}^{+}$Potential from Experimental Data. Phys. Rev. A 1994, 50, 314-316.

(10) Cencek, W.; Rychlewski, J.; Jaquet, R.; Kutzelnigg, W. SubMicrohartree Accuracy Potential Energy Surface for $\mathrm{H}_{3}^{+}$Including Adiabatic and Relativistic Effects. I. Calculation of the Potential Points. J. Chem. Phys. 1998, 108, 2831-2836.

(11) Pavanello, M.; Adamowicz, L.; Alijah, A.; Zobov, N. F.; Mizus, I. I.; Polyansky, O. L.; Tennyson, J.; Szidarovszky, T.; Császár, A. G.; Berg, M.; et al. Precision Measurements and Computations of Transition Energies in Rotationally Cold Triatomic Hydrogen Ions up to the Midvisible Spectral Range. Phys. Rev. Lett. 2012, 108, 023002.

(12) Polyansky, O. L.; Alijah, A.; Zobov, N. F.; Mizus, I. I.; Ovsyannikov, R. I.; Tennyson, J.; Lodi, L.; Szidarovszky, T.; Császár, A. G. Spectroscopy of $\mathrm{H}_{3}^{+}$Based on a New High-Accuracy Global Potential Energy Surface. Philos. Trans. R. Soc. London, Ser. A 2012, 370, 5014-5027.

(13) Adamowicz, L.; Pavanello, M. Progress in Calculating the Potential Energy Surface of $\mathrm{H}_{3}^{+}$. Philos. Trans. R. Soc. London, Ser. A 2012, 370, 5001-5013.

(14) Larsson, M. Experimental Studies of the Dissociative Recombination of $\mathrm{H}_{3}^{+}$. Philos. Trans. R. Soc. London, Ser. A 2000, 358, 2433-2444.

(15) Larsson, M. Dissociative Recombination of $\mathrm{H}_{3}^{+}$: 10 Years in Retrospect. Philos. Trans. R. Soc. London, Ser. A 2012, 370, 5118-5129.

(16) McCall, B. J.; Huneycutt, A. J.; Saykally, R. J.; Djuric, N.; Dunn, G. H.; Semaniak, J.; Novotny, O.; Al-Khalili, A.; Ehlerding, A.; Hellberg, F.; et al. Dissociative Recombination of Rotationally Cold $\mathrm{H}_{3}^{+}$. Phys. Rev. A 2004, 70, 052716.

(17) Kreckel, H.; Motsch, M.; Mikosch, J.; Glosík, J.; Plašil, R.; Altevogt, S.; Andrianarijaona, V.; Buhr, H.; Hoffmann, J.; Lammich, L.; et al. High-Resolution Dissociative Recombination of Cold $\mathrm{H}_{3}^{+}$and First Evidence for Nuclear Spin Effects. Phys. Rev. Lett. 2005, 95, 263201.

(18) Fonseca dos Santos, S.; Kokoouline, V.; Greene, C. H. Dissociative Recombination of $\mathrm{H}_{3}^{+}$in the Ground and Excited Vibrational States. J. Chem. Phys. 2007, 127, 124309.

(19) Tom, B. A.; Zhaunerchyk, V.; Wiczer, M. B.; Mills, A. A.; Crabtree, K. N.; Kaminska, M.; Geppert, W. D.; Hamberg, M.; af Ugglas, M.; Vigren, E.; et al. Dissociative Recombination of Highly Enriched para$\mathrm{H}_{3}^{+}$. J. Chem. Phys. 2009, 130, 031101.

(20) Kreckel, H.; Novotný, O.; Crabtree, K. N.; Buhr, H.; Petrignani, A.; Tom, B. A.; Thomas, R. D.; Berg, M. H.; Bing, D.; Grieser, M.; et al. High-Resolution Storage-Ring Measurements of the Dissociative Recombination of $\mathrm{H}_{3}^{+}$Using a Supersonic Expansion Ion Source. Phys. Rev. A 2010, 82, 042715 . 
(21) Varju, J.; Hejduk, M.; Dohnal, P.; Jílek, M.; Kotrík, T.; Plašil, R.; Gerlich, D.; Glosík, J. Nuclear Spin Effect on Recombination of $\mathrm{H}_{3}^{+}$Ions with Electrons at 77 K. Phys. Rev. Lett. 2011, 106, 203201.

(22) Petrignani, A.; Altevogt, S.; Berg, M. H.; Bing, D.; Grieser, M.; Hoffmann, J.; Jordon-Thaden, B.; Krantz, C.; Mendes, M. B.; Novotný, O.; et al. Resonant Structure of Low-Energy $\mathrm{H}_{3}^{+}$Dissociative Recombination. Phys. Rev. A 2011, 83, 032711.

(23) Dohnal, P.; Hejduk, M.; Varju, J.; Rubovič, P.; Roučka, S.; Kotrík, T.; Plašil, R.; Glosík, J.; Johnsen, R. Binary and Ternary Recombination of para- $\mathrm{H}_{3}^{+}$and ortho- $\mathrm{H}_{3}^{+}$with Electrons: State Selective Study at 77-200 K. J. Chem. Phys. 2012, 136, 244304.

(24) Herbst, E.; Klemperer, W. The Formation and Depletion of Molecules in Dense Interstellar Clouds. Astrophys. J. 1973, 185, 505534.

(25) Watson, W. D. The Rate of Formation of Interstellar Molecules by Ion-Molecule Reactions. Astrophys. J. 1973, 183, L17-L20.

(26) Geballe, T. R.; Oka, T. Detection of $\mathrm{H}_{3}^{+}$in Interstellar Space. Nature 1996, 384, 334-335.

(27) McCall, B. J.; Geballe, T. R.; Hinkle, K. H.; Oka, T. Detection of $\mathrm{H}_{3}^{+}$in the Diffuse Interstellar Medium Toward Cygnus OB2 No. 12. Science 1998, 279, 1910-1913.

(28) McCall, B. J.; Geballe, T. R.; Hinkle, K. H.; Oka, T. Observations of $\mathrm{H}_{3}^{+}$in Dense Molecular Clouds. Astrophys. J. 1999, 522, 338-348.

(29) Drossart, P.; Maillard, J.-P.; Caldwell, J.; Kim, S. J.; Watson, J. K. G.; Majewski, W. A.; Tennyson, J.; Miller, S.; Atreya, S. K.; Clarke, J. T.; et al. Detection of $\mathrm{H}_{3}^{+}$on Jupiter. Nature 1989, 340, 539-541.

(30) Geballe, T. R.; Jagod, M.-F.; Oka, T. Detection of $\mathrm{H}_{3}^{+}$Infrared Emission Lines in Saturn. Astrophys. J. 1993, 408, L109-L112.

(31) Trafton, L. M.; Geballe, T. R;; Miller, S.; Tennyson, J.; Ballester, G. E. Detection of $\mathrm{H}_{3}^{+}$from Uranus. Astrophys. J. 1993, 405, 761-766.

(32) Indriolo, N.; Geballe, T. R.; Oka, T.; McCall, B. J. $\mathrm{H}_{3}^{+}$in Diffuse Interstellar Clouds: A Tracer for the Cosmic-Ray Ionization Rate. Astrophys. J. 2007, 671, 1736-1747.

(33) Indriolo, N.; McCall, B. J. Investigating the Cosmic-Ray Ionization Rate in the Galactic Diffuse Interstellar Medium through Observations of $\mathrm{H}_{3}^{+}$. Astrophys. J. 2012, 745, 91 .

(34) Goto, M.; McCall, B. J.; Geballe, T. R.; Usuda, T.; Kobayashi, N.; Terada, H.; Oka, T. Absorption Line Survey of $\mathrm{H}_{3}^{+}$toward the Galactic Center Sources I. GCS 3-2 and GC IRS3. Publ. Astron. Soc. Jpn. 2002, 54, 951-961.

(35) Goto, M.; Usuda, T.; Nagata, T.; Geballe, T. R.; McCall, B. J.; Indriolo, N.; Suto, H.; Henning, T.; Morong, C. P.; Oka, T. Absorption Line Survey of $\mathrm{H}_{3}^{+}$toward the Galactic Center Sources. II. Eight Infrared Sources within $30 \mathrm{pc}$ of the Galactic Center. Astrophys. J. 2008, 688, 306-319.

(36) Quack, M. Detailed Symmetry Selection Rules for Reactive Collisions. Mol. Phys. 1977, 34, 477-504.

(37) Oka, T. Nuclear Spin Selection Rules in Chemical Reactions by Angular Momentum Algebra. J. Mol. Spectrosc. 2004, 228, 635-639.

(38) Park, K.; Light, J. C. Microcanonical Statistical Study of orthopara Conversion in the Reaction $\mathrm{H}_{3}^{+}+\mathrm{H}_{2} \rightarrow\left(\mathrm{H}_{5}^{+}\right)^{*} \rightarrow \mathrm{H}_{3}^{+}+\mathrm{H}_{2}$ at Very Low Energies. J. Chem. Phys. 2007, 126, 044305.

(39) Hugo, E.; Asvany, O.; Schlemmer, S. $\mathrm{H}_{3}^{+}+\mathrm{H}_{2}$ Isotopic System at Low Temperatures: Microcanonical Model and Experimental Study. J. Chem. Phys. 2009, 130, 164302.

(40) Crabtree, K. N.; Tom, B. A.; McCall, B. J. Nuclear Spin Dependence of the Reaction of $\mathrm{H}_{3}^{+}$with $\mathrm{H}_{2}$. I. Kinetics and Modeling. J. Chem. Phys. 2011, 134, 194310.

(41) Gómez-Carrasco, S.; González-Sánchez, L.; Aguado, A.; SanzSanz, C.; Zanchet, A.; Roncero, O. Dynamically Biased Statistical Model for the ortho/para Conversion in the $\mathrm{H}_{3}^{+}+\mathrm{H}_{2} \rightarrow \mathrm{H}_{3}^{+}+\mathrm{H}_{2}$ Reaction. J. Chem. Phys. 2012, 137, 094303.

(42) Cordonnier, M.; Uy, D.; Dickson, R. M.; Kerr, K. E.; Zhang, Y.; Oka, T. Selection Rules for Nuclear Spin Modifications in Ion-Neutral Reactions Involving $\mathrm{H}_{3}^{+}$. J. Chem. Phys. 2000, 113, 3181-3193.

(43) Crabtree, K. N.; Kauffman, C. A.; Tom, B. A.; Beçka, E.; McGuire, B. A.; McCall, B. J. Nuclear Spin Dependence of the Reaction of $\mathrm{H}_{3}^{+}$with $\mathrm{H}_{2}$. II. Experimental Measurements. J. Chem. Phys. 2011, 134, 194311.
(44) Grussie, F.; Berg, M. H.; Crabtree, K. N.; Gärtner, S.; McCall, B. J.; Schlemmer, S.; Wolf, A.; Kreckel, H. The Low-Temperature Nuclear Spin Equilibrium of $\mathrm{H}_{3}^{+}$in Collisions with $\mathrm{H}_{2}$. Astrophys. J. 2012, 759, 21.

(45) Crabtree, K. N.; Indriolo, N.; Kreckel, H.; Tom, B. A.; McCall, B. J. On the ortho:para Ratio of $\mathrm{H}_{3}^{+}$in Diffuse Molecular Clouds. Astrophys. J. 2011, 729, 15.

(46) Millar, T. J.; Bennett, A.; Herbst, E. Deuterium Fractionation in Dense Interstellar Clouds. Astrophys. J. 1989, 340, 906-920.

(47) Roberts, H.; Herbst, E.; Millar, T. J. The Importance of New Rate Coefficients for Deuterium Fractionation Reactions in Interstellar Chemistry. Mon. Not. R. Astron. Soc. 2002, 336, 283-290.

(48) Pagani, L.; Vastel, C.; Hugo, E.; Kokoouline, V.; Greene, C. H.; Bacmann, A.; Bayet, E.; Ceccarelli, C.; Peng, R.; Schlemmer, S. Chemical Modeling of L183 (L134N): An Estimate of the ortho/para $\mathrm{H}_{2}$ Ratio. Astron. Astrophys. 2009, 494, 623-636.

(49) Bunker, P.; Jensen, P. Molecular Symmetry and Spectroscopy, 2nd ed.; NRC Publications: Ontario, 1998.

(50) The groups $S_{3}$ and $S_{3}^{*}$ are called $C_{3 v}(M)$ and $D_{3 h}(M)$, respectively, in ref 49.

(51) Strictly speaking, the electronic symmetry should also be included when considering the allowed combinations of rovibrational (rovibronic) and nuclear spin wave functions. For $\mathrm{H}_{3}^{+}$, however, there are no observed stable electronically excited states, and the ground electronic state is totally symmetric, meaning that it has no practical effect on the symmetry rules discussed.

(52) Landau, L. D.; Lifshitz, E. M. Quantum Mechanics: Non-Relativistic Theory, 2nd ed.; Pergamon Press: New York, 1965; pp 97-100.

(53) McCall, B. J. Spectroscopy of $\mathrm{H}_{3}^{+}$in Laboratory and Astrophysical Plasmas. Ph.D. thesis, University of Chicago, 2001.

(54) Above the barrier to linearity, symmetric top basis states no longer adequately describe the rotational structure of $\mathrm{H}_{3}^{+}$, but these states will not be treated here. Thus, this analysis is applicable in the limit that $J, k$, and $l$ are all good quantum numbers.

(55) Hougen, J. T. Classification of Rotational Energy Levels for Symmetric-Top Molecules. J. Chem. Phys. 1962, 37, 1433-1441.

(56) Application of the symmetry projectors to different direct product basis states gives linear combinations that vary by only a phase factor.

(57) Hugo, E. The $\mathrm{H}_{3}^{+}+\mathrm{H}_{2}$ Isotopic System: Origin of Deuterium Astrochemistry. Ph.D. thesis, Universität zu Köln, Germany, 2008.

(58) Oka, T.; Epp, E. The Nonthermal Rotational Distribution of $\mathrm{H}_{3}^{+}$. Astrophys. J. 2004, 613, 349-354.

(59) Kreckel, H.; Petrignani, A.; Berg, M.; Bing, D.; Reinhardt, S.; Altevogt, S.; Buhr, H.; Froese, M.; Hoffmann, J.; Jordon-Thaden, B.; et al. Electron Collisions and Rovibrational Action Spectroscopy of Cold $\mathrm{H}_{3}^{+}$Molecules. J. Phys.: Conf. Ser. 2007, 88, 012064.

(60) Widicus Weaver, S. L.; Woon, D. E.; Ruscic, B.; McCall, B. J. Is $\mathrm{HO}_{2}^{+}$a Detectable Interstellar Molecule? Astrophys. J. 2009, 697, 601609 . 